Louisiana State University

LSU Digital Commons

Faculty Publications

Department of Physics \& Astronomy

$12-8-2008$

\title{
Low energy gamma-ray emission from Galactic black holes
}

\author{
Gary L. Case \\ Louisiana State University \\ Michael L. Cherry \\ Louisiana State University \\ James C. Ling \\ Jet Propulsion Laboratory \\ William A. Wheaton \\ California Institute of Technology
}

Follow this and additional works at: https://digitalcommons.Isu.edu/physics_astronomy_pubs

\section{Recommended Citation}

Case, G., Cherry, M., Ling, J., \& Wheaton, W. (2008). Low energy gamma-ray emission from Galactic black holes. AIP Conference Proceedings, 1053, 145-152. https://doi.org/10.1063/1.3009473

This Conference Proceeding is brought to you for free and open access by the Department of Physics \& Astronomy at LSU Digital Commons. It has been accepted for inclusion in Faculty Publications by an authorized administrator of LSU Digital Commons. For more information, please contact ir@lsu.edu. 


\title{
Low Energy Gamma-Ray Emission from Galactic Black Holes
}

\author{
Gary L. Case*, Michael L. Cherry*, James C. Ling ${ }^{\dagger}$ and William A. \\ Wheaton** \\ *Louisiana State University, Baton Rouge, LA 70803 USA \\ ${ }^{\dagger}$ Jet Propulsion Laboratory, California Institute of Technology, Pasadena, CA 91109 USA \\ ${ }^{* *}$ Infrared Processing and Analysis Center, California Institute of Technology, Pasadena, CA \\ 91125 USA
}

\begin{abstract}
X-ray observations of Galactic black holes (GBHs) such as Cygnus X-1 have greatly advanced the understanding of these objects. However, the vast majority of the observations have been restricted to energies below $\sim 200 \mathrm{keV}$. The Compton Gamma-Ray Observatory (CGRO) allowed for the first time simultaneous observations at energies from $\sim 25 \mathrm{keV}$ up to $>1 \mathrm{GeV}$. In particular, the BATSE experiment aboard $C G R O$ was able to monitor low-energy gamma-ray emission from Cygnus X-1, as well as other GBHs, nearly continuously over a nine year period. Using the Enhanced BATSE Occultation Package (EBOP), light curves and spectra in the energy range $25-2000 \mathrm{keV}$ have been obtained for six GBHs. Based on the spectra when the GBHs were in a high gamma-ray flux state, it is suggested that at least two different classes of GBHs exist. The first is characterized by a Comptonization spectrum below $\sim 200 \mathrm{keV}$ followed by a soft power law excess as exhibited by Cygnus X-1, GRO J0422+32, GRO J1719-24, and GX 339-4. The second class is characterized by simple power law spectrum in the full $25-2000 \mathrm{keV}$ range, with no evidence for a Comptonization component, as exhibited by GRO J1655-40 and GRS 1915+105.

Gamma-ray observations can serve as an important diagnostic in studying the physical processes around GBHs. More sensitive observations in the future at energies $>250 \mathrm{keV}$ will help answer questions regarding issues such as the nonthermal electron distribution, state transitions, and the connection to jets.
\end{abstract}

Keywords: Gamma-rays, Galactic Black Holes, Cygnus X-1, GRO J0422+32, GRO J1719-24, GX 339-4, GRO J1655-40, GRS 1915+105

PACS: $95.85 . \mathrm{Pw}$, 97.60.Lf, 97.80.Jp

\section{INTRODUCTION}

Much of what is known today about the physical processes occurring in the vicinity of Galactic black holes (GBHs) has come from observations made in x-rays. These objects are bright when viewed in X-rays, and over the last several decades there has been a large number of x-ray instruments, from rockets to balloons to satellites, that have obtained good quality data. The first GBH candidate, Cygnus X-1, was discovered by a rocket experiment in the 1960s [1]. Balloon-borne experiments led to characterizations of its spectral shape [2,3], while space-based experiments discovered its flux state transitions $[4,5,6]$, and later, its spectral state transitions [7]. Early gamma-ray observations showed that Cygnus X-1 is variable at higher energies $[8,9,10]$, with episodic emission observed up to $>1 \mathrm{MeV}[11,12]$.

Other GBH candidates were discovered in X-rays, dramatically increasing the interest

CP1053, Observational Evidence for Black Holes in the Universe, Proceedings of the $2^{\text {nd }}$ Kolkata Conference and of the Satellite Meeting on Black Holes, Neutron Stars, and Gamma-Ray Bursts, edited by S. K. Chakrabarti and A. S. Majumdar, (C) 2008 American Institute of Physics 978-0-7354-0582-0/08/\$23.00 


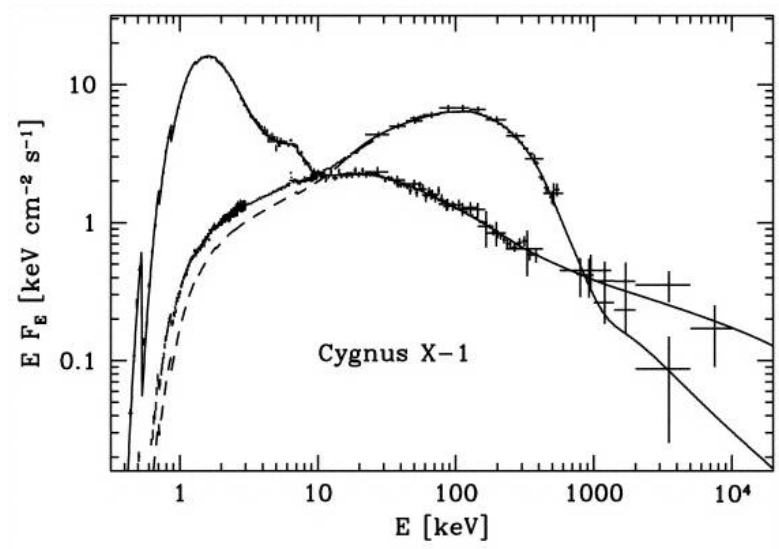

FIGURE 1. Cygnus $X-1$ spectra in the high (hard) and low (soft) gamma-ray states. Data are from BeppoSAX, BATSE, OSSE and COMPTEL, and the solid curves represent fits with the EQPAIR model. Taken from [16].

in these objects. However, the majority of the observations were restricted to energies below $\sim 200 \mathrm{keV}$ and/or to short, pointed observations. The Compton Gamma-Ray Observatory (CGRO) allowed for the first time simultaneous observations at energies from $\sim 25 \mathrm{keV}$ up to $>1 \mathrm{GeV}$. In particular, the BATSE experiment aboard $C G R O$ was able to monitor GBHs nearly continuously, using the Earth occultation technique, for a nine year period $(1991-2000)$ in the low-energy gamma-ray regime $(25-2000 \mathrm{keV})$.

The BATSE instrument was comprised of eight identical NaI detectors located on the eight corners of the spacecraft. These detectors had no direct imaging capability. It was recognized early on that the Earth occultation technique could be used to obtain fluxes for known gamma-ray sources. Two parallel efforts at NASA Marshall Space Flight Center [13, 14] and NASA JPL [15] resulted in catalogs of average fluxes, light curves and spectra for over 100 gamma-ray sources. The Enhanced BATSE Occultation Package (EBOP) developed at JPL was used to generate spectra for a number of GBHs. Results are presented showing the BATSE spectra for the GBHs Cygnus X-1, GRO J0422+32, GRO J1719-24, GX 339-4, GRO J1655-40, and GRS 1915+105.

\section{CYGNUS X-1}

During the CGRO era, Cygnus X-1 was in a high gamma-ray state about $90 \%$ of the time, corresponding to the canonical $\mathrm{x}$-ray low/hard state. In this hard state, the soft $\mathrm{x}$ ray emission (below $10 \mathrm{keV}$ ) is suppressed, and the peak of the energy emission occurs at $\sim 100 \mathrm{keV}$. The data above $20 \mathrm{keV}$ are best fit by a model with both a Comptonization component and a soft power law tail at high energies. Figure 1 shows the hard state spectra, with data from the CGRO instruments BATSE, OSSE, and COMPTEL as well as BeppoSAX, fitted with the hybrid EQPAIR model [17].

Twice between 1991 and 2000, Cygnus X-1 made a flux state transition. A transition 

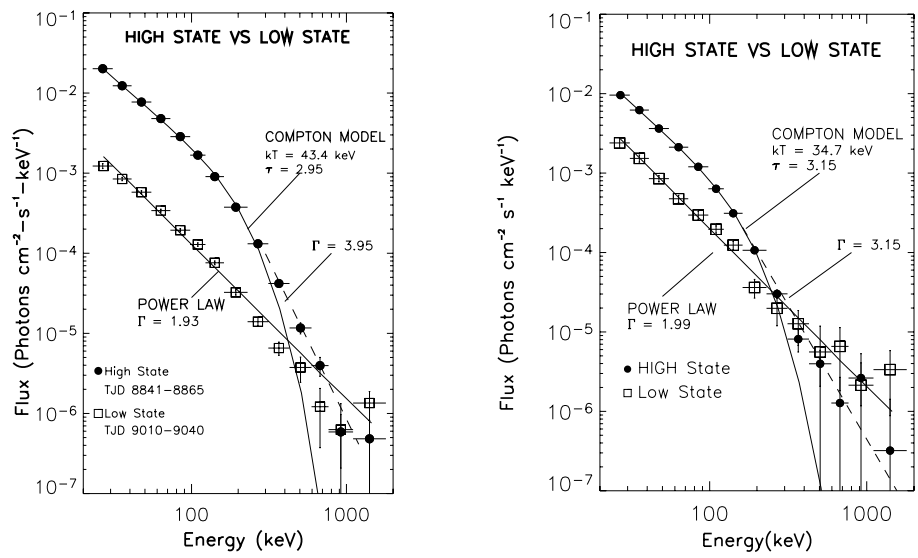

FIGURE 2. High and low gamma-ray state spectra of GRO J0422+32 (left panel) and GRO J171924 (right panel). Both sources show Comptonization spectra below about $200 \mathrm{keV}$ with a soft power law excess above $200 \mathrm{keV}$. Spectra for GRO J0422+32 and GRO J1719-24 taken from [20] and [21], respectively.

occurred in 1994 and then again in 1996, each lasting about 150 days. During the first transition, the low energy gamma-ray flux was observed to decrease and the spectral shape change from the typical hard state spectrum to a single power law [18]. During the second transition [19], contemporaneous observations with BeppoSAX, BATSE, and OSSE showed that the soft x-ray flux increased dramatically, while the hard x-ray/low energy gamma-ray flux again decreased. This state is referred to as the soft state. The spectrum above $\sim 30 \mathrm{keV}$ can be fit with a single power law component extending out to $>1 \mathrm{MeV}$ (see Fig. 1), with the spectral index harder than that for the hard state above $100 \mathrm{keV}$. The two spectra have a pivot point at $\sim 1 \mathrm{MeV}$, indicating a new type of anti-correlation between fluxes below and above $1 \mathrm{MeV}$, which is in contrast to the one seen in the $\mathrm{x}$-ray region $(\sim 10 \mathrm{keV})$ previously associated with the two spectral states. For more discussion, see [16]. These observations suggest that the nonthermal electron distribution is important in both the high (hard) and low (soft) gamma-ray states.

\section{GRO J0422+32, GRO J1719-24, AND GX 339-4}

GRO J0422+32 was discovered by BATSE in August 1992 when it went into outburst. It rose quickly, with the $35-100 \mathrm{keV}$ flux as measured by BATSE/EBOP staying near its maximum of $\sim 3 \mathrm{Crab}$ for $\sim 20$ days before undergoing a slow decay. The spectrum while in the high gamma-ray flux state is shown in Fig. 2 (left panel). This spectrum is best fit by a model with a Comptonization component below $\sim 300 \mathrm{keV}$ and a soft power law tail excess above $\sim 300 \mathrm{keV}$ (for more details, see [20]). This is similar to what is observed in Cygnus X-1 when it is in the hard state. About 120 days after its initial peak, GRO J0422+32 underwent a second brightening, reaching a secondary maximum at about $15 \%$ of the primary peak. The average spectra for a 30 day period during this 


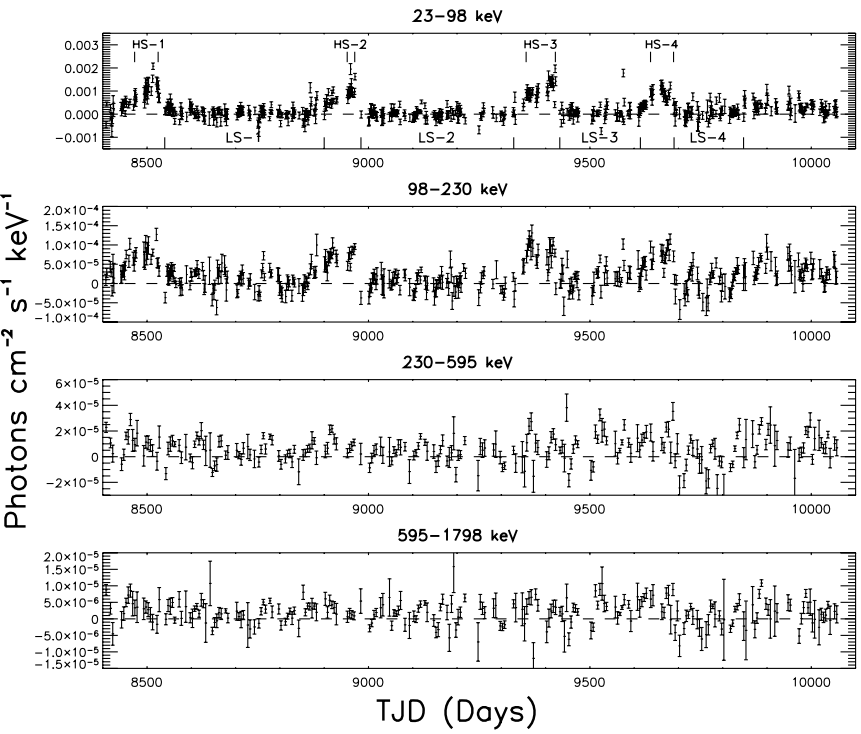

FIGURE 3. BATSE/EBOP light curve for GX 339-4 in four broad energy bands. The high and low state boundaries are marked and referenced in Fig. 4.

lower state is also shown in Fig. 2 (left panel).

GRO J1719-24 was also discovered by BATSE, as well as SIGMA, in September 1993 when it went into outburst. It also rose quickly, reaching a peak flux of 1.5 Crab in the $35-100 \mathrm{keV}$ band as measured by BATSE/EBOP. It decayed slowly to $\sim 1 \mathrm{Crab}$ over $\sim 70$ days before abruptly decreasing to less than 100 mCrab. About 260 days later, GRO J1719-24 began a series of smaller outbursts, spanning about one year, before returning to quiescence. The spectra for both the high and low gamma-ray flux states are shown in Fig. 2 (right panel). As with GRO J0422+32, the high flux state spectrum is best fit by a model with a Comptonization component below $\sim 250 \mathrm{keV}$ and a soft power law tail excess above $\sim 250 \mathrm{keV}$ (for more details, see [21]). This also is similar to what is observed in Cygnus X-1 when it is in the hard state.

GX 339-4 was observed by BATSE to undergo four outbursts starting in May 1991. All four outbursts peaked at about $400-500 \mathrm{mCrab}$ in the $23-98 \mathrm{keV}$ energy band. Each outburst remained in the high state for $\sim 55-70$ days before returning to a low state, consistent with pre-outburst flux levels. GX 339-4 remained in this quiescent state between the outbursts for 150 - 300 days. After the fourth outburst, GX 339-4 underwent a period of smaller outbursts spanning $\sim 300$ days with peak fluxes about $10 \%$ of the peaks of the previous outbursts. The BATSE/EBOP light curve for four broad energy bands is shown in Fig. 3 [22]. The light curve in the $23-98 \mathrm{keV}$ band is shown with 1-day resolution, the $98-230 \mathrm{keV}$ band is shown with 2-day resolution, and both the $230-595$ and $595-1798 \mathrm{keV}$ bands are shown with 5-day resolution.

The high gamma-ray flux states for the four outbursts have been defined in Fig. 3. Each of these high states was fit individually to a model with a Comptonization com- 

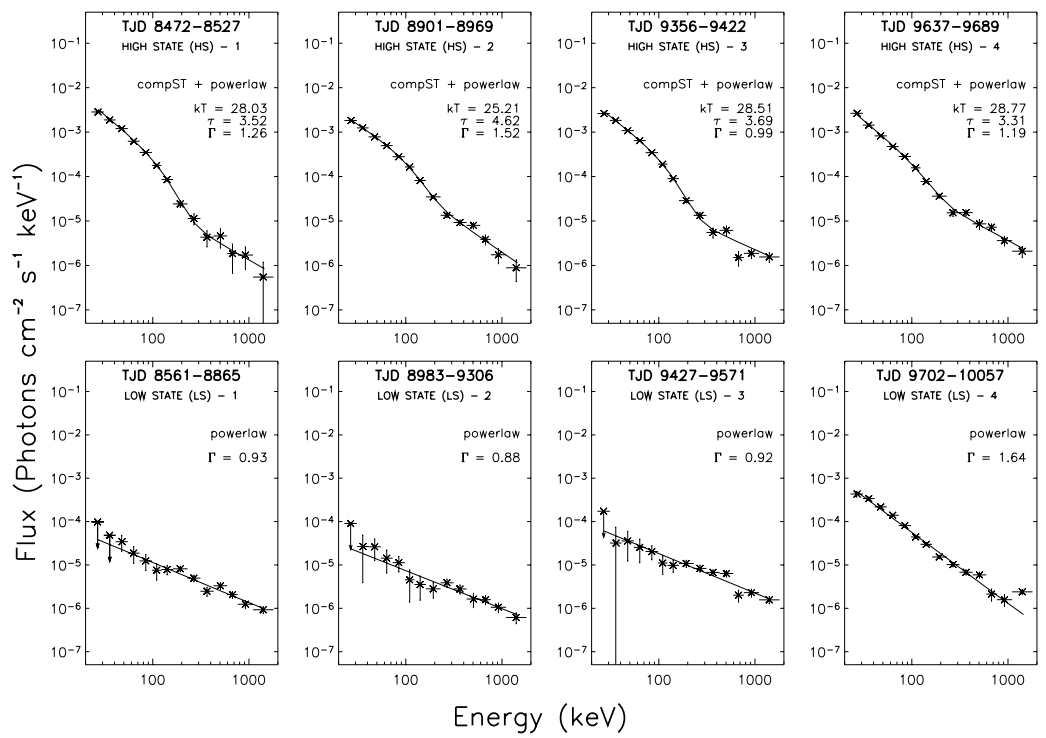

FIGURE 4. Spectra for GX 339-4 during the high (top row) and low (bottom row) gamma-ray states as defined in Fig. 3

ponent (the COMPST model in XSPEC) plus a power law. The results are shown in Fig. 4 [22]. The fit parameters are fairly consistent, with moderate plasma temperatures $k T=25-28 \mathrm{keV}$, optical depths $\tau=3.3-4.7$, and a hard power law index $\Gamma=1-1.5$. These spectra are also similar to the high flux states of Cygnus X-1, GRO J0422+32, and GRO J1719-24, albeit with slightly lower values of $k T$. It is suggested then that GX 339-4 was in a hard state when it was in outburst [22].

The bottom four panels of Fig. 4 show the spectra when GX 339-4 was in the low states as defined in Fig. 3. The first three spectra are very hard and consistent with quiescence, while the spectrum of LS-4 is statistically consistent with a steeper, though still quite hard, power law with index $\Gamma=1.6$. A Comptonization model was fit to this data, but did not yield a statistically better fit than the power law.

\section{GRO J1655-40 AND GRS 1915+105}

GRO J1655-40 was observed by BATSE to produce five large flares, with peak fluxes in the $23-98 \mathrm{keV}$ band of $>1 \mathrm{Crab}$, as well as several smaller flares [23]. Combining the data from the days when the source was in its high gamma-ray flux state yields a power law spectrum with a photon index $\Gamma=2.82$. No emission was detected above $\sim 300$ $\mathrm{keV}$ to the sensitivity limit of BATSE/EBOP (Fig. 5 left panel). This is consistent with the photon index $\Gamma=2.76$ reported by OSSE from the sum of 31 days of observations made during portions of the first four flares [24]. Spectra from BATSE/EBOP for GRO J1655-40 (as well as GRS 1915+105) were first presented in [23]. However, the data 

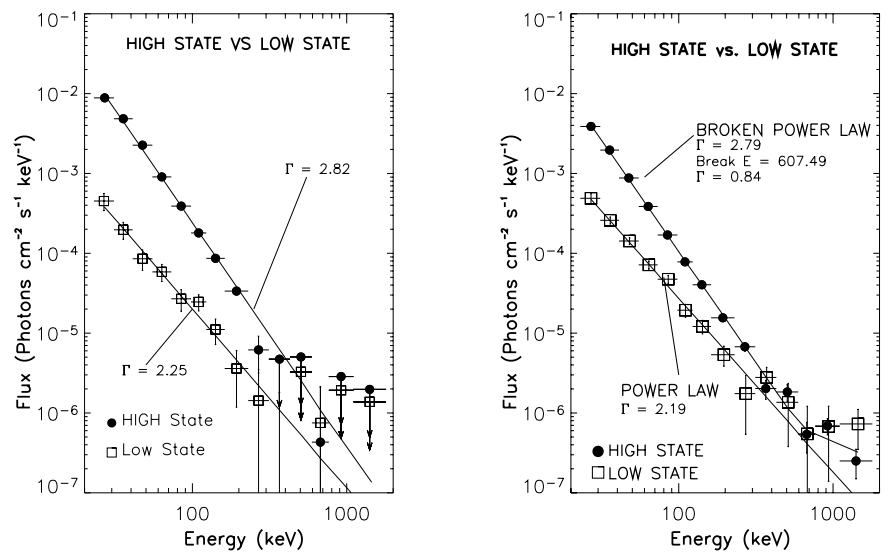

FIGURE 5. High and low gamma-ray flux state spectra of GRO J1655-40 (left panel) and GRS $1915+105$ (right panel).

have now been reprocessed, with the results in Fig. 5 (left panel) consistent with those of [23]. In the low gamma-ray flux state, the GRO J1655-40 spectrum is best fit by a power law with a hard index of $\Gamma=2.25$ with no significant emission above $\sim 300 \mathrm{keV}$.

GRS 1915+105 was extremely active throughout the entire 9-year lifetime of CGRO $[14,23]$, though the peak fluxes in the $23-98 \mathrm{keV}$ band were much lower than GRO J1655-40. Combining the data from when GRS 1915+105 was in high gamma-ray flux state yields a spectrum best fit by a broken power law, with low-energy spectral index $\Gamma=2.79$ hardening to $\Gamma=0.8$ at higher energies, with a break energy of $\sim 600 \mathrm{keV}$ (Fig. 5 right panel). Note that significant emission is seen out beyond $300 \mathrm{keV}$. As noted by [23], this high-energy emission appears to be variable. In the low gamma-ray flux state, the GRS 1915+105 spectrum is best fit by a power law with a hard index of $\Gamma=2.19$, similar to what is obtained for GRO J1655-40, though GRS 1915+105 shows emission beyond $300 \mathrm{keV}$, even in the low gamma-ray flux state.

\section{DISCUSSION}

When Cygnus $\mathrm{X}-1$ is in the hard state, the gamma-ray spectrum is characterized by a Comptonization component and a soft power law excess above the Comptonization component. The hard x-ray/low energy gamma-ray emission can be described by the reprocessing of the soft photons from the disk by a hot electron corona by means of inverse Compton scattering. However, the power law component at higher energies suggests the presence of a nonthermal electron distribution. When Cygnus X-1 made the transition to the soft state, the soft $\mathrm{x}$-ray flux increased dramatically and the hard $\mathrm{x}$-ray/low energy gamma-ray flux decreased. The gamma-ray spectrum in this soft state is characterized by a power law that extends out beyond $1 \mathrm{MeV}$, also suggesting the presence of nonthermal electrons.

The three GBHs GRO J0422+32, GRO J1719-24, and GX 339-4 were observed to 
undergo significant gamma-ray outbursts during the $C G R O$ lifetime. All three sources rose from quiescence to high gamma-ray flux states, with the BATSE/EBOP spectra for all three sources resembling the Cygnus X-1 hard state spectrum. When in a low gammaray state, all three sources show hard power law spectra. Because the behavior of these GBHs as exhibited by their gamma-ray spectra is similar to Cygnus X-1, it is suggested that that all three of these GBHs were in the hard state during their outbursts.

The GBHs GRO J1655-40 and GRS 1915+105 also were observed to undergo gamma-ray outbursts. However, when these GBHs were in a high gamma-ray flux state, their spectra showed only a power law component, with a relatively hard spectral index. No Comptonization component is evident in these spectra. The spectrum for GRO J1655-40 cuts off $\sim 300 \mathrm{keV}$, while the spectrum of GRS 1915+105 extends to at least $500 \mathrm{keV}$. These spectra are consistent with the Cygnus X-1 spectrum in the soft state. This suggests that when these two GBHs went into outburst, they were in the soft state.

Many models have been proposed to explain the hard and soft states of GBHs. The canonical model consists of a cool, thin accretion disk with a hot corona or Compton cloud. Hard x-rays/low energy gamma-rays are produced by inverse Compton scattering of the disk photons by the corona. When the accretion rate increases, the disk emission increases and the corona is quenched. The two states of Cygnus $\mathrm{X}-1$, as well as the outbursts of GRO J0422+32 and GRO J1719-24, have been modeled successfully with an advection-dominated accretion flow (ADAF) in the x-ray region [25]. However, this model cannot account for the excess emission seen above $\sim 200 \mathrm{keV}$. Two-component flows have been proposed (e.g. [26]) that take into account the converging flow and the Comptonization that can occur due to the bulk motion of the material falling onto the black hole. But these models also do not reproduce the observed emission above $\sim 200 \mathrm{keV}$. It has been suggested that the strong episodic MeV emission [12] with weak evidence for a correlated narrow annihilation line [27] observed by HEAO-3 could arise from a high temperature $(k T \approx 400 \mathrm{keV})$, pair-dominated plasma very near the black hole [28]. However, this has not been observationally validated.

Hybrid models such as EQPAIR [17, 29] that incorporate both thermal (coronal) and nonthermal electron distributions have been used to model emission from a few $\mathrm{keV}$ to greater than $1 \mathrm{MeV}$ (see Fig. 1). But these models are more phenomenological and do not have a physical basis for the origin of the nonthermal electrons. A more recent model $[30,31]$ combines the accretion flow with the converging flow to produce a shock region that forms at the centifugal barrier supported boundary layer (CENBOL) surrounding the black hole. This region naturally gives rise to the nonthermal electrons, which could produce the $>200 \mathrm{keV}$ emission by Compton upscattering the soft $\mathrm{x}$ ray photons from the disk or Compton downscattering the high energy gamma-ray photons produced near the black hole e.g. in the jet. This CENBOL region could also be responsible for driving the jets themselves [32]. The gamma-ray emission could also be produced by Componization of synchrotron photons generated by the nonthermal electrons. Low energy gamma-ray observations could address questions such as: What are the characteristics of the nonthermal electron population? Why are some GBHs going into outburst in a soft state while others are in a hard state? What role do jets play in the high energy emission? The CENBOL model will be applied to the gamma-ray data from BATSE/EBOP to try and constrain the emission mechanisms of the gammaray emission in both classes of GBHs (those like Cygnus X-1, GRO J0422+32, GRO 
J1719-24, and GX 339-4, and those like GRO J1655-40 and GRS 1915+105) and investigate the connection to the $\mathrm{x}$-ray observations. Future sensitive low energy gammaray observations will be an important tool in continuing to understand the fundamental processes occurring in GBHs.

\section{ACKNOWLEDGMENTS}

The authors wish to acknowledge the contribution of Robert Radocinski in the development of the EBOP software. LSU students C. Britt, C. Fannin, J. Hostetter, J. Rodi, and C. Welch, and Caltech students V. Aguilar and D. Wells, participated in analyzing the BATSE data. This work was supported in part by NASA award NAG5-10253 and NASA/Louisiana Board of Regents grant NNX07AT62A/LEQSF(2007-10) Phase3-02.

\section{REFERENCES}

1. S. Bowyer, et al., Science 147, 394-398 (1965).

2. R. C. Haymes, and F. R. Harnden, Astroph. J. 159, 1111-1114 (1970).

3. R. A. Sunyaev, and L. G. Titarchuk, Astron. and Astroph. 86, 121-138 (1980).

4. H. Tananbaum, et al., Astroph. J. 177, L5-L10 (1972).

5. J. Heise, et al., Nature 256, 107-108 (1975).

6. S. S. Holt, E. A. Boldt, L. J. Kaluzienski, and P. J. Serlemitsos, Nature 256, 108-109 (1975).

7. J. F. Dolan, et al., Nature 267, 813-815 (1977).

8. J. L. Matteson, R. F. Mushotzky, W. S. Paciesas, and J. G. Laros, "Intensity and spectral variations of CYG X-1 observed from balloons," in X-ray Binaries, NASA Goddard Space Flight Center, 1976, pp. 407-424.

9. P. L. Nolan, et al., Astroph. J. 246, 494-501 (1981).

10. M. L. McConnell, et al., Astroph. J. 343, 317-322 (1989).

11. R. E. Baker, R. R. Lovett, K. J. Orford, and D. Ramsden, Nature Physical Science 245, 18-19 (1973).

12. J. C. Ling, W. A. Mahoney, W. A. Wheaton, and A. S. Jacobson, Astroph. J. 321, L117-L122 (1987).

13. B. A. Harmon, et al., Astroph. J. Suppl. 138, 149-183 (2002).

14. B. A. Harmon, et al., Astroph. J. Suppl. 154, 585-622 (2004).

15. J. C. Ling, et al., Astroph. J. Suppl. 127, 79-124 (2000).

16. M. L. McConnell, et al., Astroph. J. 572, 984-995 (2002).

17. P. S. Coppi, "The Physics of Hybrid Thermal/Non-Thermal Plasmas," in High Energy Processes in Accreting Black Holes, edited by J. Poutanen, and R. Svensson, 1999, vol. 161 of Astronomical Society of the Pacific Conference Series, pp. 375-403.

18. J. C. Ling, et al., Astroph. J. 484, 375-382 (1997).

19. S. N. Zhang, et al., Astroph. J. 477, L95-L98 (1997).

20. J. C. Ling, and W. A. Wheaton, Astroph. J. 584, 399-413 (2003).

21. J. C. Ling, and W. A. Wheaton, Astroph. J. 622, 492-502 (2005).

22. J. C. Ling, et al., Astroph. J. p. in preparation (2008).

23. G. L. Case, et al., Chinese J. of Astron. and Astroph. Suppl. 5, 341-346 (2005).

24. J. E. Grove, et al., Astroph. J. 500, 899-908 (1998).

25. A. A. Esin, et al., Astroph. J. 505, 854-868 (1998).

26. K. Ebisawa, L. Titarchuk, and S. K. Chakrabarti, Pub. of the Astron. Soc. of Japan 48, 59-65 (1996).

27. J. C. Ling, and W. A. Wheaton, Astroph. J. 343, L57-L59 (1989).

28. E. P. Liang, and C. D. Dermer, Astroph. J. 325, L39-L42 (1988).

29. M. Gierlinski, et al., Monthly Notices of the Royal Astronomical Society 309, 496-512 (1999).

30. S. K. Chakrabarti, and L. G. Titarchuk, Astroph. J. 455, 623-639 (1995).

31. S. K. Chakrabarti, and S. Mandal, Astroph. J. 642, L49-L52 (2006).

32. S. K. Chakrabarti, Astron. and Astroph. 351, 185-191 (1999). 